\title{
Prevalence of intestinal parasitic infections among HIV/AIDS patients from two health institutions in Abuja, Nigeria
}

\author{
Abaver $\mathrm{DT}^{1}$, Nwobegahay $\mathrm{JM}^{2},{ }^{*}$ Goon $\mathrm{DT}^{3}$, Iweriebor $\mathrm{BC}^{2}$, Anye $\mathrm{DN}^{4}$
}

1. Department of Biological Sciences, University of Abuja, Abuja, Nigeria

2. Department of Microbiology, University of Venda, Thohoyandou, South Africa

3. Centre for Biokinetics, Recreation and Sport Science, University of Venda, Thohoyandou, South Africa

4. Institute of Agricultural Research and Development (IRAD) Nkolbission, Ministry of Scientific Research and Innovation, Yaounde, Cameroon

\begin{abstract}
Background: Intestinal parasitic infections play a vital role in the prognosis of HIV/AIDS in patients.

Objectives: The aim of this study was to determine the prevalence of intestinal parasitic infections (IPIs) in HIV-infected individuals in two health facilities in Abuja-Nigeria.

Methods: A cross sectional study was conducted in two sites: the GEDE AIDS and Infectious Diseases Research Institute (GAIDRI), and the Human Virology Institute-General Hospital Asokoro-Abuja, Nigeria. A total of 119 subjects were recruited (85 HIV infected and $34 \mathrm{HIV}$ negative). Stool specimens collected were analyzed macroscopically and microscopically for consistency and the presence of enteric parasites.

Results: The overall prevalence rate of enteroparasites obtained in this study was $22.7 \%(27 / 119)$. The prevalence of intestinal parasitic infections in HIV infected patients was $24.7 \%$; while in HIV negative persons, it was 17.6\%. However, the high rate obtained for HIV infected patients was not statistically significant ( $>$ > 0.05).

Conclusion: Although the prevalence rate of enteric parasites in HIV/AIDS patients was higher than in HIV negative individuals, this difference is not statistically significant. Even though there was no statistical difference in the two groups, parasitic infections in HIV/AIDS patients often result in debilitating illness.
\end{abstract}

Keywords: Prevalence; Parasitic infection; Acquired Immunodeficiency Syndrome

African Health Sciences 2011; 11(S1): S24 - S27

\section{Introduction}

The HIV/AIDS pandemic is a major public health crisis facing the world today. An estimated 33.0 (30.3 -36.2) million adults and children are living with the virus worldwide ${ }^{1}$. Sub-Saharan Africa is one of the regions that have been hardest hit by this pandemic; with an estimated $22(20.5-23.6)$ million adults and children living with the virus in this part of the world ${ }^{1}$. Nigeria is one of the countries with the highest prevalence rate in the region. In Nigeria, it was estimated $^{2}$ that 2.9-3.3 million people were living with HIV/AIDS by the end of 2005, with about 220, 000 deaths from AIDS and 930,000 AIDS orphans ${ }^{3}$. In a similar manner, intestinal parasitic infections are

*Corresponding author

Daniel T Goon

Centre for Biokinetics, Recreation and Sport Science

University of Venda

X5050 Thohoyandou

South Africa

E-mail: daniel goon@,univen.ac.za widely distributed throughout the world, especially in sub-Saharan Africa. In this region, these infections are responsible for about 1 million deaths each year ${ }^{4}$. Furthermore HIV/AIDS significantly weakens the immunity of the body, rendering itsusceptible to various opportunistic parasitic infections.

The lack of safe, portable drinking water, poor hygienic and sanitary conditions are largely responsible for the spread of intestinal parasites in most African countries. Parasites frequently associated with acute and chronic diarrhea in HIV disease include various species of Cryptosporidium spp, Isospora belli, Microsporidia spp, Giardia intestinalis, Entamoeba spp, Cyclospora cayetanensis, Blastocystis, Strongyloides, and Ascarisc lumbricoides. ${ }^{5}$. Intestinal parasitic infections create a huge concern in the management and care of HIV positive people. Detecting these parasites and understanding the status and significance of the infections they cause will greatly help in proper management and treatment of these patients. 
However, few studies ${ }^{6,7}$ have addressed the issue of parasitic infections in Nigeria. These studies were concentrated in the South-West and South-South regions of Nigeria. There is hardly available information on the prevalence of intestinal parasitic infections in the Central-Northern part of Nigeria. Information on the prevalence of intestinal parasitic infections in an area can help public health bodies to better check further spread within the general population. Such data underlie the necessity of early diagnosis and management in order to prevent the health complications among HIV/AIDS patients. Therefore, this study was carried out to examine the prevalence of intestinal parasitic infections among HIV/AIDS patients in two health facilities in Abuja, Nigeria.

\section{Methods}

\section{Patients and study area}

In this cross sectional study, a total of 119 participants were recruited from among the outpatients in two health institutions in Nigeria (GEDE AIDS and Infectious Diseases Research Institute Maitama (GAIDRI) and the Human Virology InstituteGeneral Hospital Asokoro-Abuja). The mean age of the participants was 29.2 years, ranging from 1 51 years. The proportion of females and males was $50.4 \%$ and $49.6 \%$ respectively. The study participants were briefed on the nature and purpose of the study and participation was voluntary. They were also assured of anonymity for their laboratory testing, after oral consent was obtained from them.

\section{Sample collection}

Samples were collected between October 2005 and March 2006. Two mls of venous blood was collected by a trained phlebotomist from a total of one hundred and nineteen (94 from Asokoro and 25 from GAIDRI) consenting participants. All blood samples were screened for the presence of HIV antibody using the Smart Check HIV $1 / 2^{8}$. Reactive samples were confirmed using the Western blood assay $^{5}$. The $\mathrm{CD}^{+}{ }^{+}$counts were determined manually?
Values were enumerated only for those with evidence of HIV infection ( $\mathrm{n}=85$ ).

About $2 \mathrm{~g}$ of stool samples were collected from all the participants using sterile universal bottles. They were examined macroscopically and microscopically for color, consistency and the presence of ova, larvae, trophozoites and cysts of intestinal parasites. Briefly, $1 \mathrm{~g}$ of faeces was mixed with $10 \%$ formol saline, treated with ether and centrifuged. Smears were made from the pellets, airdried, fixed with methanol and examined under the microscope. Morphological features were taken into consideration in the identification of the different parasites.E.histolytica was distinguished from other Entamoeba spp. by observing red blood cells ingested by the trophozoite stage of the parasite.

\section{Ethical considerations}

The Ethical Committee of the University of Abuja approved the study design and research. Permission for this study was obtained from the GAIDRI and the Human Virology Institute-General Hospital Asokoro-Abuja, Nigeria. Informed consent was obtained from all study participants before they were enrolled in the study.

\section{Data analysis}

Demographic and other data were analyzed using Statistical Package for Social Sciences (SPSS version 17.0; Chicago, USA). Significant differences between categorical variables were determined using the independent-samples t-test. A p value of $<0.05$ was considered statistically significant.

\section{Results}

The prevalence of intestinal parasitic infections was $24.7 \%$ in HIV infected patients and $17.6 \%$ in HIV negative persons (Table 1). However, the high rate obtained for HIV infected patients was not statistically significant $(95 \% \mathrm{CI}=0.976-0.670 \mathrm{df}$ $=117, \mathrm{p}>0.05)$. The overall prevalence rate of enteroparasites obtained in this study was $22.7 \%$ (27/ 119). Loose and watery stools were observed in $21.8 \%(26 / 119)$ of the subjects.

Table 1: The prevalence of intestinal parasites in the two sites

\begin{tabular}{lccccc}
\hline Site & \multicolumn{2}{l}{ HIV seropositive } & & HIV seronegative & \\
\cline { 2 - 3 } & $\begin{array}{c}\text { Number } \\
\text { examined }\end{array}$ & $\begin{array}{c}\text { Enteroparasites } \\
\text { Number }(\%)\end{array}$ & & Number examined & $\begin{array}{c}\text { Enteroparasites } \\
\text { Number }(\%)\end{array}$ \\
\hline Asokoro Hospital & 70 & $10(14.3 \%)$ & & 24 & $2(8 \%)$ \\
GAIDRI & 15 & $11(73.3 \%)$ & & 10 & $4(40 \%)$ \\
Total & 85 & $21(24.7 \%)$ & 34 & $6(17.6 \%)$ \\
\hline
\end{tabular}

GAIDRI = GEDE AIDS and Infectious Diseases Research Institute 
The parasites detected were Entamoeba histolytica (6.7\%), Hookworm (4.2\%), Ascaris lumbricoides (2.5\%), Entamoeba coli $(2.5 \%)$, Giardia lamblia (2.5\%), Strongyloides stercoralis $(2.5 \%)$, Trichiura trichura $(0.8 \%)$ and Taenia spp. (0.8\%).
Four different parasites namely; Entamoeba coli, Giardia lamblia, Strongyloides stercoralis, and Trichiura trichura $(0.8 \%)$ were detected only in HIV seropositive individuals (Table 2).

Table 2: Prevalence of intestinal parasitic infections in HIV positive and HIV negative persons

\begin{tabular}{llll}
\hline Type of parasite found & $\begin{array}{c}\text { HIV infected } \\
(\mathrm{n}=85)\end{array}$ & $\begin{array}{c}\text { HIV negative } \\
(\mathrm{n}=34)\end{array}$ & $\begin{array}{c}\text { Total }(\%) \\
(\mathrm{n}=119)\end{array}$ \\
\hline Entamoeba histolytica & 6 & 2 & $8(6.7 \%)$ \\
Hookworm & 3 & 2 & $5(4.2 \%)$ \\
Ascaris lumbricoides & 1 & 2 & $3(2.5 \%)$ \\
Entamoeba coli & 3 & 0 & $3(2.5 \%)$ \\
Giardia lamblia & 3 & 0 & $3(2.5 \%)$ \\
Strongyloides stercoralis & 3 & 0 & $3(2.5 \%)$ \\
Trichuris trichura & 1 & 0 & $1(0.8 \%)$ \\
Taenia spp. & 1 & 0 & $1(0.8 \%)$ \\
Total number of parasites & 21 & 6 & $27(13.6$ \\
\hline 95\%CI=0.976-0.670, $\mathrm{p}=0.576$ & &
\end{tabular}

\section{Discussion}

Intestinal parasite infections have enormous consequences on the health of HIV/AIDS patients. Sub-Sahara Africa is already over burdened by human immunodeficiency virus (HIV) infection and chronic diarrhea due to intestinal parasites is also common in this region ${ }^{5,6,7}$. Enteric pathogens are frequently transmitted by unhygienic habits and cause morbidity and mortality. Several species of protozoa and other intestinal parasites have been associated with acute and chronic diarrhea, and even weight loss in HIV/AIDS patients.

The overall prevalence rate of enteroparasites obtained in this study was $22.7 \%$. Of HIV sero positive persons $21 / 85(24.7 \%)$ had intestinal parasites as well as of $6 / 34(17.6 \%)$ of seronagative individuals. However, this difference is not statistically significant $(\mathrm{p}>0.05)$. The parasites detected were E. histolytica (6.7\%), Hookworm (4.2\%), A. lumbricoides (2.5\%), E. coli (2.5\%), G. lamblia (2.5\%), Strongyloides stercoralis $(2.5 \%)$, T. trichura $(0.8 \%)$ and Taenia spp. $(0.8 \%)$ Four different parasites namely; Entamoeba coli, G. lamblia, S. stercoralis, and T. trichura were detected only in HIV serpositive individuals.

In the present study, the most prevalent parasite detected in HIV/AIDS patients was E. histolytica. This is in conformity with previous studies done in Abuja by Udeh et al. ${ }^{10}$ where the highest prevalence obtained for this parasite was $36.79 \%$. Infection with E. histolytica can lead to amoebic dysentery or amoebic liver abscess in severe cases. The higher rate of this parasite infection in Abuja metropolis seems quite amazing and worrisome. The geographical location of Abuja, which is in a tropical S26 region, may offer a plausible explanation. The survival of ova of most intestinal helminthes and cysts of protozoans is favorably guaranteed in a tropical environment. Provision of portable drinking water, sanitary facilities and health education on hygienic practices will go a long way in reducing the incidence of parasitic infections in the general population

These parasites have been reported among HIV/AIDS patients in sub-Saharan African countries. In 2008, Mariam et al..$^{5}$ found $11.5 \%$ of S. stercoralis in HIV/AIDS patients in Ethiopia. Studies have also reported the presence of other intestinal parasites in HIV/AIDS patients in other parts of Nigeria ${ }^{6,11,12}$.

\section{Conclusion}

We have highlighted the presence of various intestinal parasites among HIV-infected patients in Nigeria. The results obtained in this study could provide vital information on various types of enteric parasites circulating among HIV/AIDS patients in Abuja, hence their relevance in the management of these patients.

\section{Acknowledgements}

We thank the authorities of GEDE AIDS and Infectious Diseases Research Institute Maitama (GAIDRI), and the Human virology InstituteGeneral Hospital Asokoro-Abuja, Nigeria for permitting us to use their facilities. The cooperation of the patients is also appreciated.

African Health Sciences Vol 11 Special Issue 1 August 2011 


\section{References}

1. UNAIDS/WHO AIDS epidemic update. Geneva, Switzerland, 2008.

2. Federal Ministry of Health (FMOH). National $\mathrm{HIV} /$ syphilis sero-prevalence sentinel survey among pregnant women attending antenatal clinics. Abuja, 2005.

3. UNAID/WHO. Report on the global AIDS epidemic. Geneva, Switzerland, 2006

4. WHO Expert Committee. Prevention and control of schistosomiasis and soil transmitted helminthiasis. WHO technical report series 2002; 912:1-57.

5. Mariam ZT, Abebe G, Mulu A. Opportunistic and other intestinal parasitic infections in AIDS patients, HIV seropositive healthy carriers and HIV seronegative individuals in Southwest Ethiopia. E. Afr. J. Pub. Health 2008; 5(3): 169172.

6. Okodua M, Adeyeba OA, Tatfeng YM, Okpala HO. Age and sex distribution of intestinal parasites infections among HIV infected subjects in
Abeokuta, Nigeria. Online J. Health Allied Sci. 2003; 4:3-5.

7. Awogun IA. The prevalence of intestinal parasitic infection in children living in Ilorin, Kwara State, Nigeria. West Afr. J. Med. 1984; 4(1):16-21.

8. GLOBALEMED, LLC. 1101 King Street, suite 370 Alexandria, VA 22314-299.

9. Schmidt RE. Monoclonal Antibodies for diagnosis of immunodeficiencies. Ann. Haematol. 1989; 59(3):200-206.

10. Udeh EO, Goselle ON, Popova D, Abelau M, Popov TV, Jean N, David JS. The prevalence of intestinal protozoans in HIV/AIDS patients in Abuja, Nigeria. Science World J. 2008; 3(3):1-4.

11. Olusegun AF, Okaka CE, Machado RLD. Isosporiasis in HIV/AIDs patients in Edo state Nigeria. Mal. J. Med. Sci. 2009; 16(3): 43-46.

12. Adesiji YO, Lawal RO, Taiwo SS, Fayemiwo SA, Adeyeba OA. Cryptosporidiasis in HIV infected patients with diarrhoea in Osun state Southwestern Nigeria. Eur. J. Gen. Med. 2007; 4(3):119-122. 